This article has been scanned by iThenticat No plagiarism detected

Volume 3, Issue 4, August 2021

p. 371-381

\title{
DESIGN VARIABLES FOR THE USE OF DIGITAL MODELING IN CONTEMPORARY INTERIOR DESIGN
}

http://dx.doi.org/10.47832/2757-5403.4-3.32

Wisam Hassan HASHIM1

\begin{abstract}
:
The current research highlights an important aspect of the great and rapid development in the field of science and technology, and modern manufacturing methods as a result of the scientific revolution resulting from the accelerated cognitive development, Which prompted designers in general and interior design, the subject of research in particular, to exploit and invest in digital technology, And the development of digital control in the industrial product design process for the purpose of creativity and innovation through these digital programs, Digital models fulfill the requirements and desires of the interior designer according to the creative skill by using modern software with high efficiency and accuracy in line with the requirements of the user, the interior designer and the beneficiary companies, Therefore, the research problem can be determined by the following question: What is the nature of the variables in modeling patterns in the stages of contemporary interior space design through the use of virtual reality techniques?

While the aim of this study was: to reveal the importance of digital models in the real interior space through a virtual reality, the current research included three sections: The first topic: the variables and their relationships in the design of the interior space, and the second topic: modeling in interior design between reality and simulation, and the third topic: Digital models in interior design, and the research reached a set of conclusions, the most important of which are:

1.Digital models allow the designer, custodians, and people to be able to explore the interior space before designing it.

2.Designers use Digital Prototyping to design, improve, validate and digitally visualize their spaces throughout the design stages.

3. Innovative digital models can be created through creative designs, achieving multiple design goals (best performance, high efficiency, space efficiency, appropriate design cost).
\end{abstract}

Key words: Variable, Digital Prototyping, Interior Design.

\footnotetext{
${ }^{1}$ Dr. , Baghdad University, Iraq, wisamalmuhamade@gmail.com, https://orcid.org/0000-0002-0330-4662
} 
المتغيرات التصميمية لاستخدام النمذجة الرقمية في التصميم الداخلي المعاصر

وسنام حسن هاشم 2

يسلط البحث الحالي الضوء على جانب مهم من جوانب التطور الكبير في مجال العلم

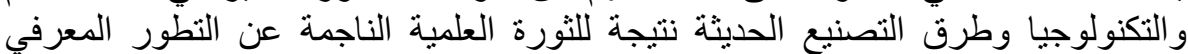

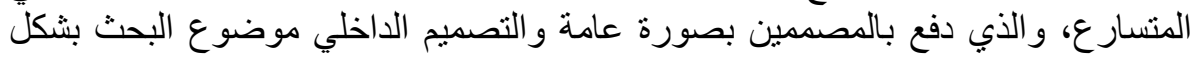

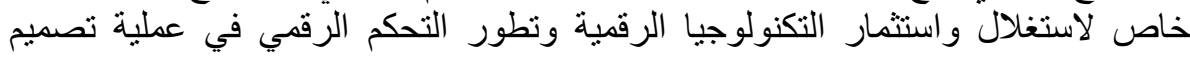

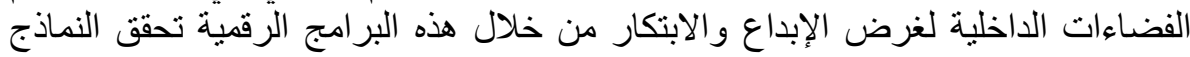

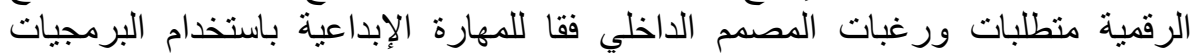
الحديثة بكفاءة عالية ودقة متتاهية تتسجم ومنطابات المستخدم و المصمم الداخلي والثركات لالترات المستفيدة.

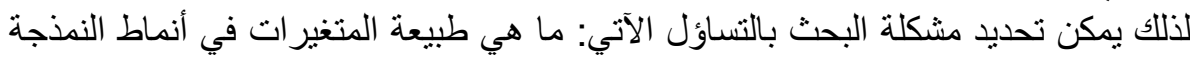

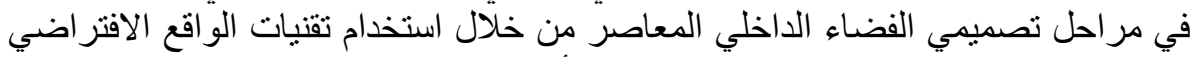

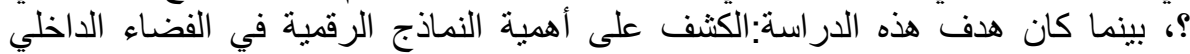

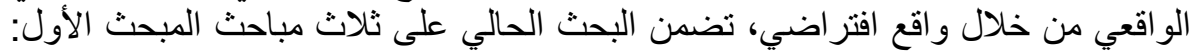

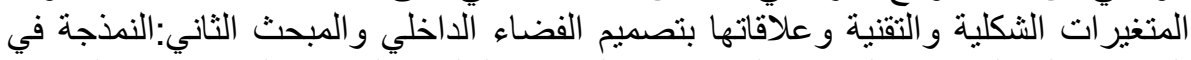

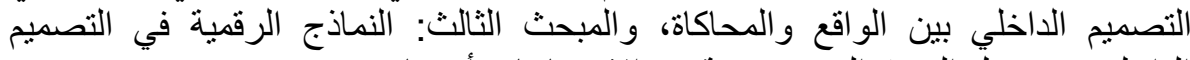

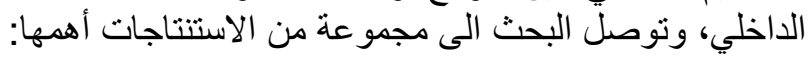

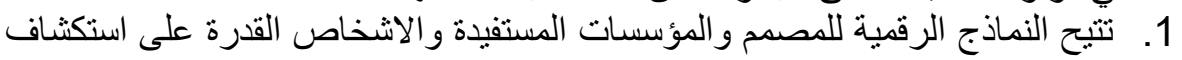
الفضاء الداخلي قبل تصميمه. 2. يستخدم المصمدون Digital Prototyping لتصميم فضائاتهم وتحسينها والتحقق منها وتصور ها رقميًا طو ال مر احل التصميج.

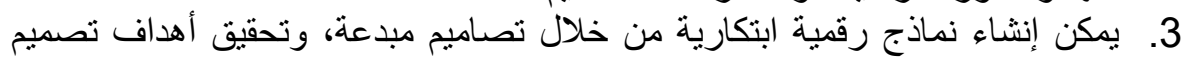

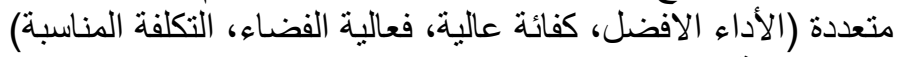

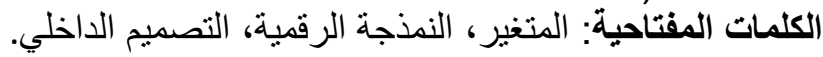

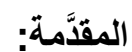

يعتبر اختبار مخرجات العملية التصميمة والتحقق بمدى تحقيقها الأهداف ومعالجة الاخفاقات أو التطوير على التصميم

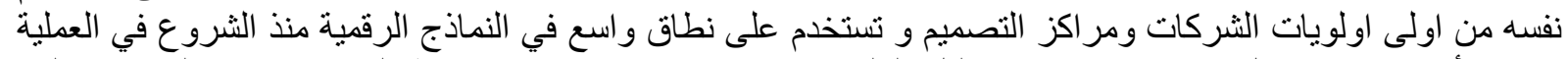

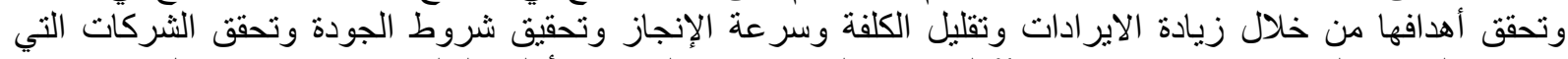

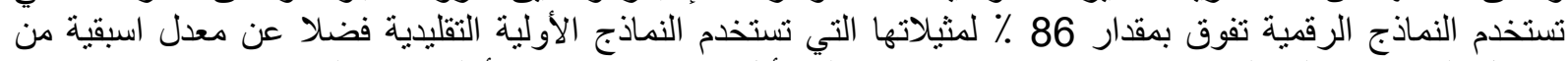

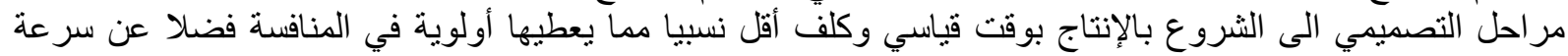

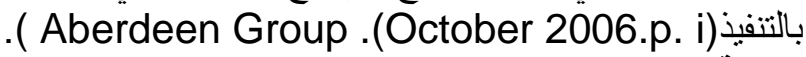

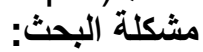

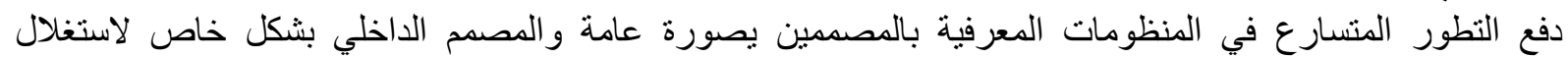

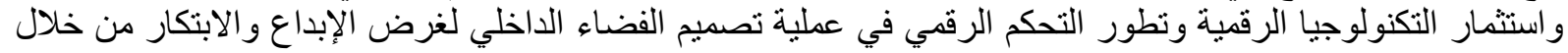

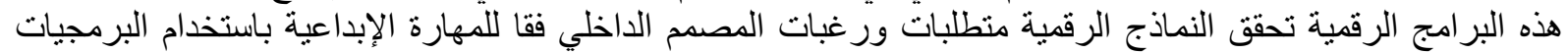

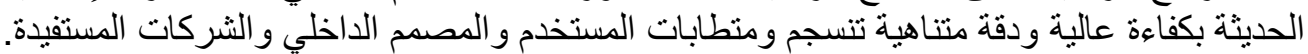

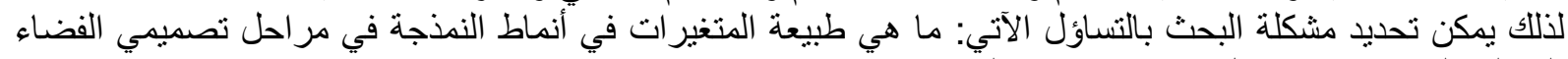

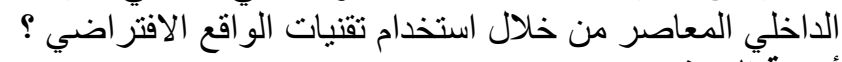

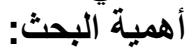




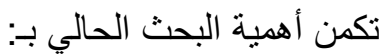

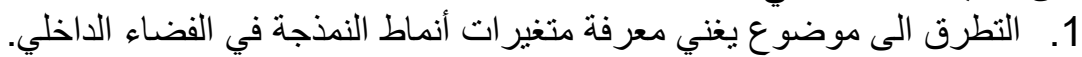

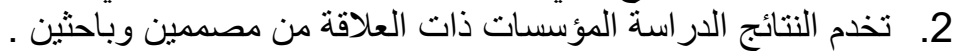

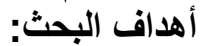

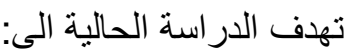

$$
\begin{aligned}
& \text { الكثفف على أهمية النماذج الرقية الكية في الفضاء الداخلي الو اقعي من خلال و اقع افتراضي .حدود البحث: }
\end{aligned}
$$

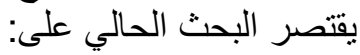

$$
\begin{aligned}
& \text { المتغير ات الحاصلة على العملية التصميمية الفضاءات الداخلية المصممة افتر اضيا، عام } 2021
\end{aligned}
$$

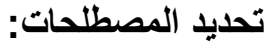

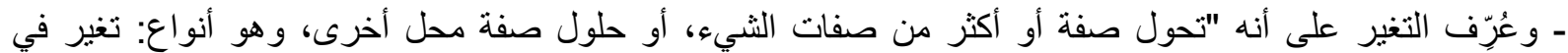

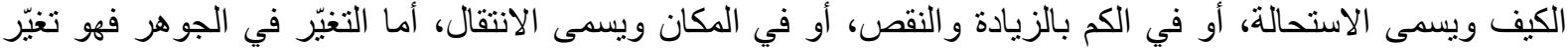

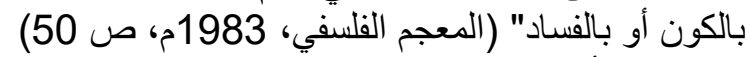

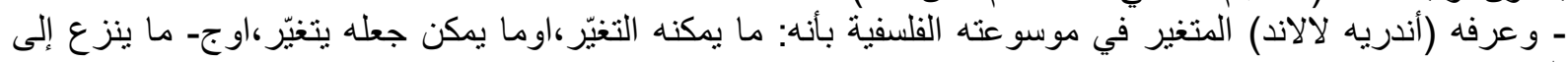

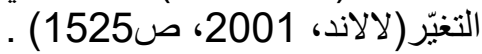

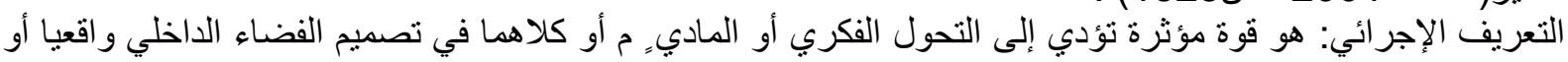

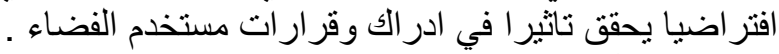

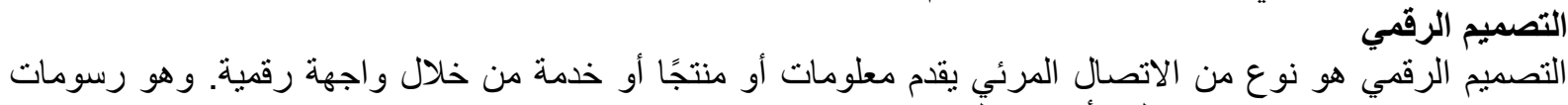

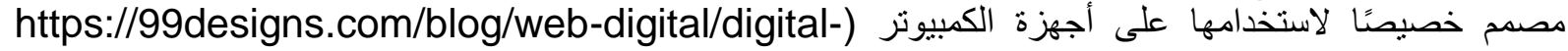

(/design عرفها الباحث اجر ائيا: بانه الثقنية الذي يتم من خلالها تصميم فضاءات افتراضية باستخدام البرامج التصميمة ومن خلال

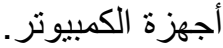

(النمذجة (Prototype):

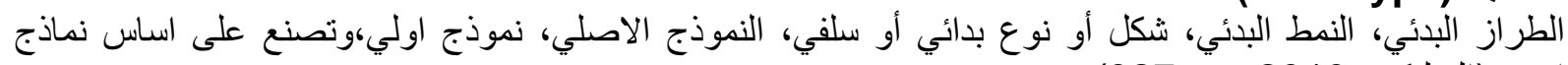

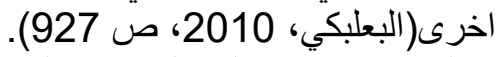

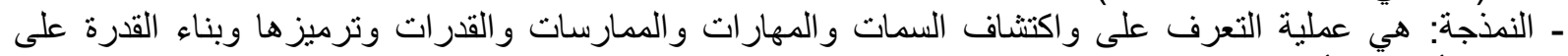

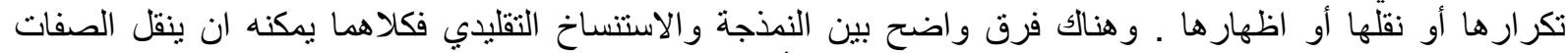

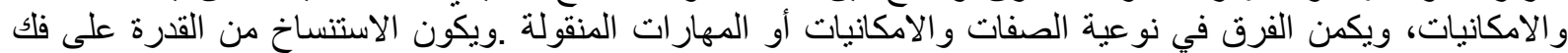

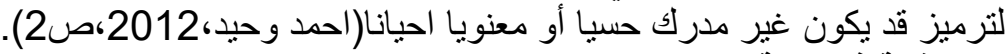

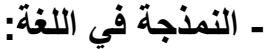

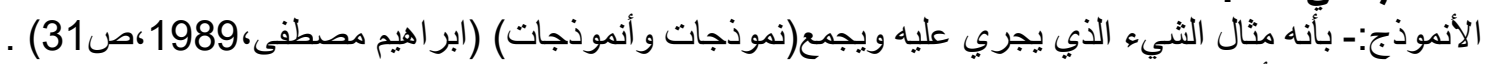

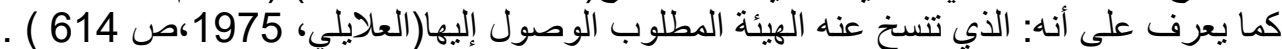

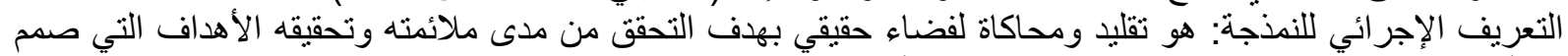
من أجلها، فضلا عن الإجراني للكوين انطباع مسبق لمستخدم أو المستفيد.

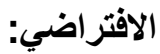
عرف بانه: بعني تصور تفاعلي ذآتي التوجيه متعدد المسارات تتنجه نظم الحاسوب الالي يعرض لنا شكل اصطناعي

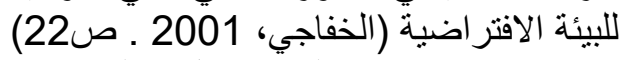

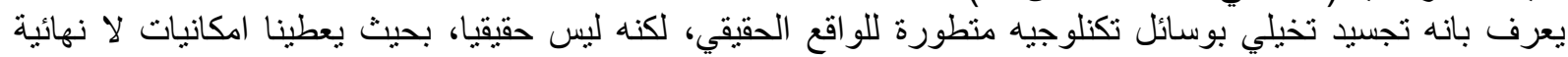

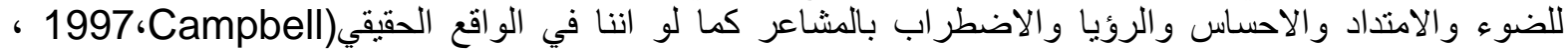
.). (p.126 التعريف الإجر ائي للافتراض: هو محاورة فكرية يؤسساها المصمم الداخلي لقصدية نقل مستخدم الفضاء الداخلي قبل

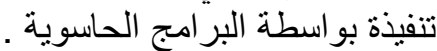

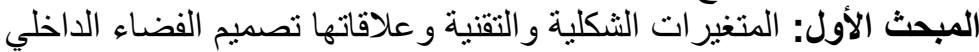

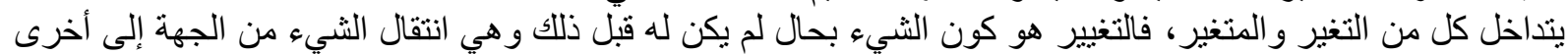

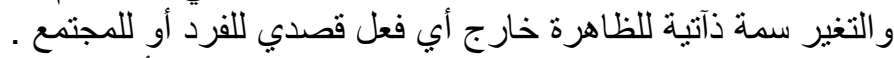

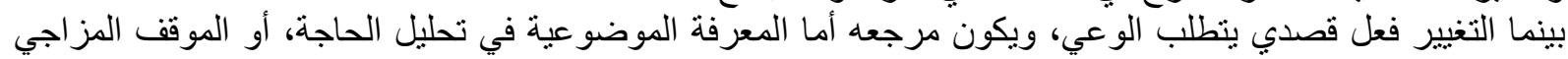
المتفرد الداعي إلى التميز فحسب، ويمكن التمييز بين عمليتي التغير والتغيير، فئي حين يمثل التوازن بين التينة الحاجة 
و التكنولوجيا التغير غير القصدي، يمثل الفرد التغيير القصدي من خلال مصدرين للتغيير هما: المعرفة الموضوعية

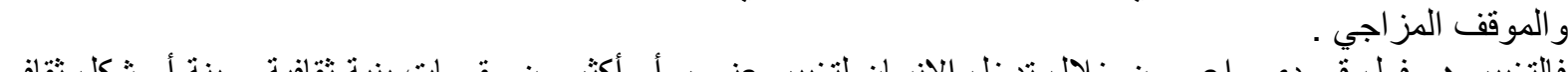
فالتغيير هو فعل قصدي والفي واعي من خلال تدخل الإنسان لتغيير عنصر أو أكثر من مقومات بنية ثقافية معينة أو شكل ثقافي

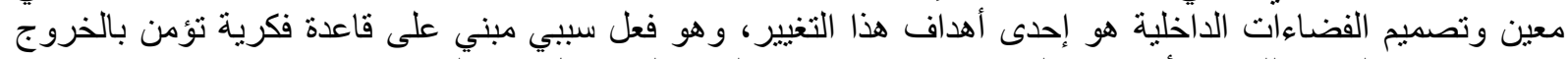

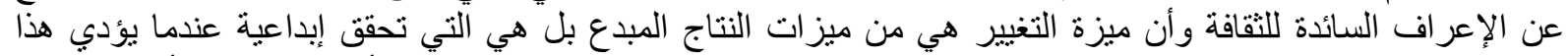

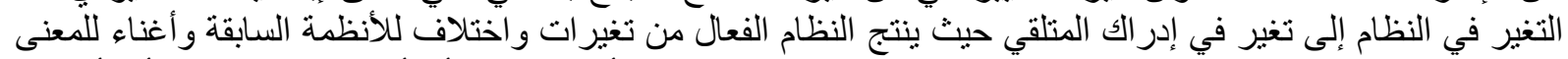

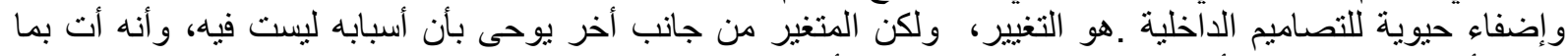

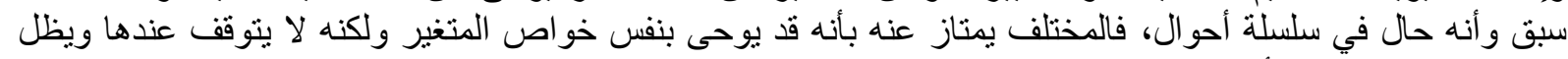

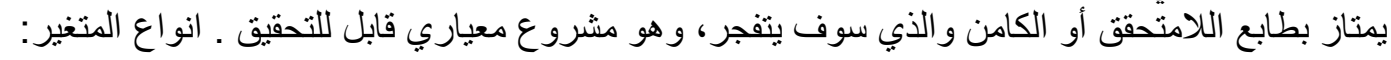
•

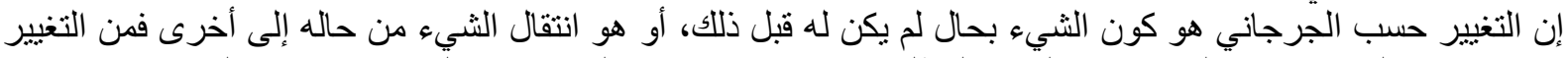

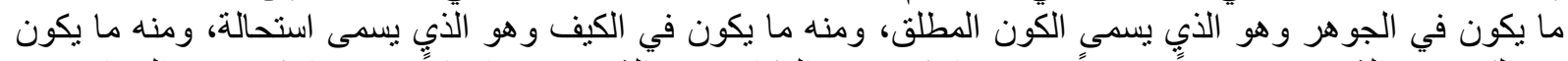

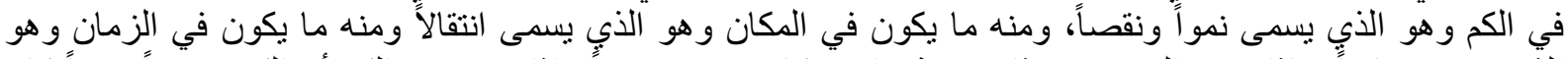

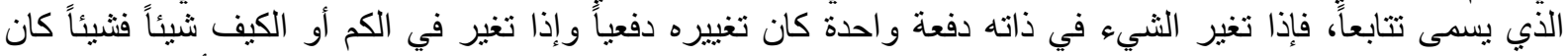

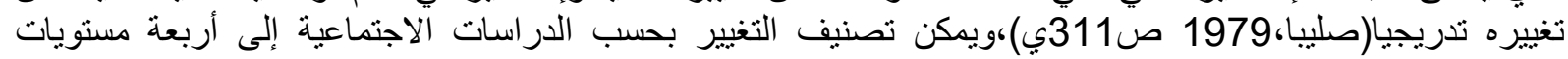

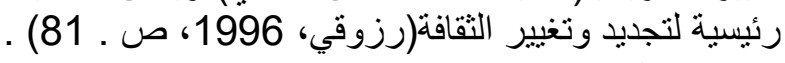

Variation النتوية
•

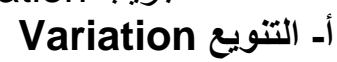

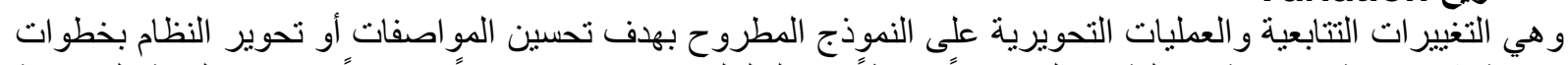

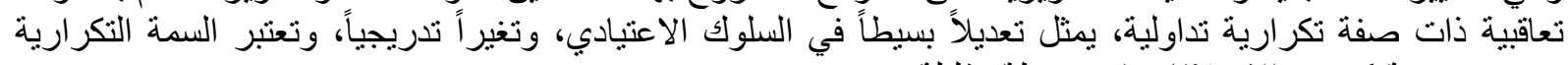

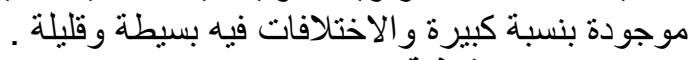
ب- الاستعارة الثقافية Cultural borrowing

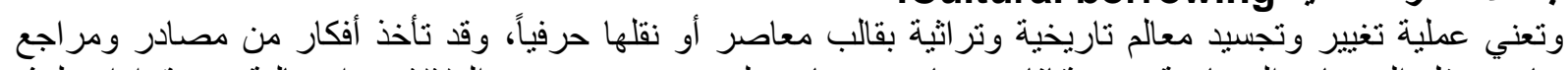

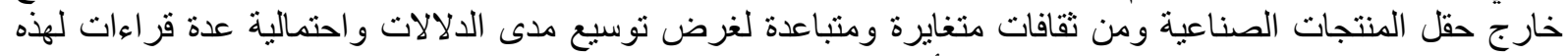

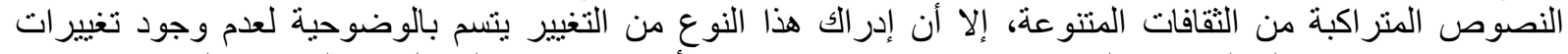

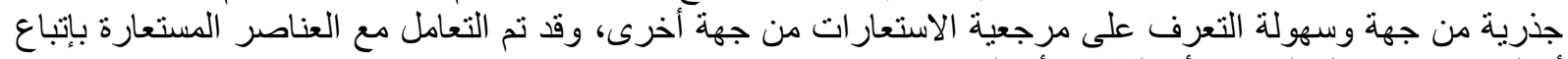
أساليب متنو عة مثل التر اكبة أو أو الطباقية أو التكثيف .

ج- الاختراع Invention

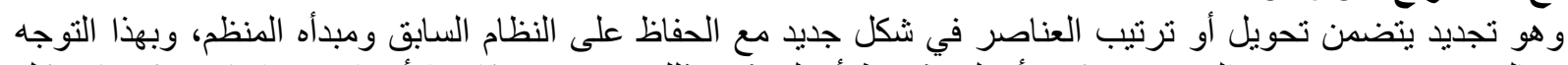

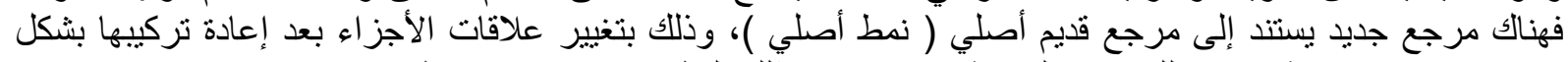

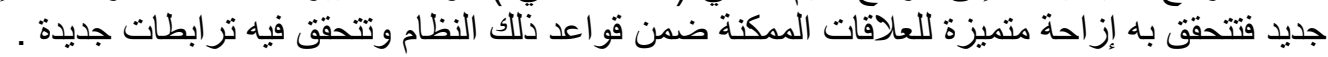

د- دالتجريب Temptation:

و هو أخر مستوى من مستويات التغيير والذي يتم فيه خرق الأعراف السائدة وتحدي القوانين الرئيسية للنظام، ويتم فيه

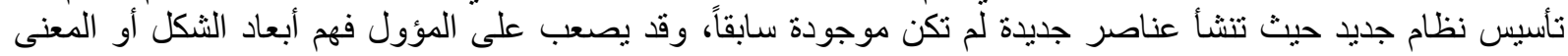

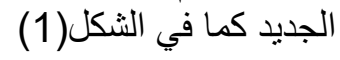




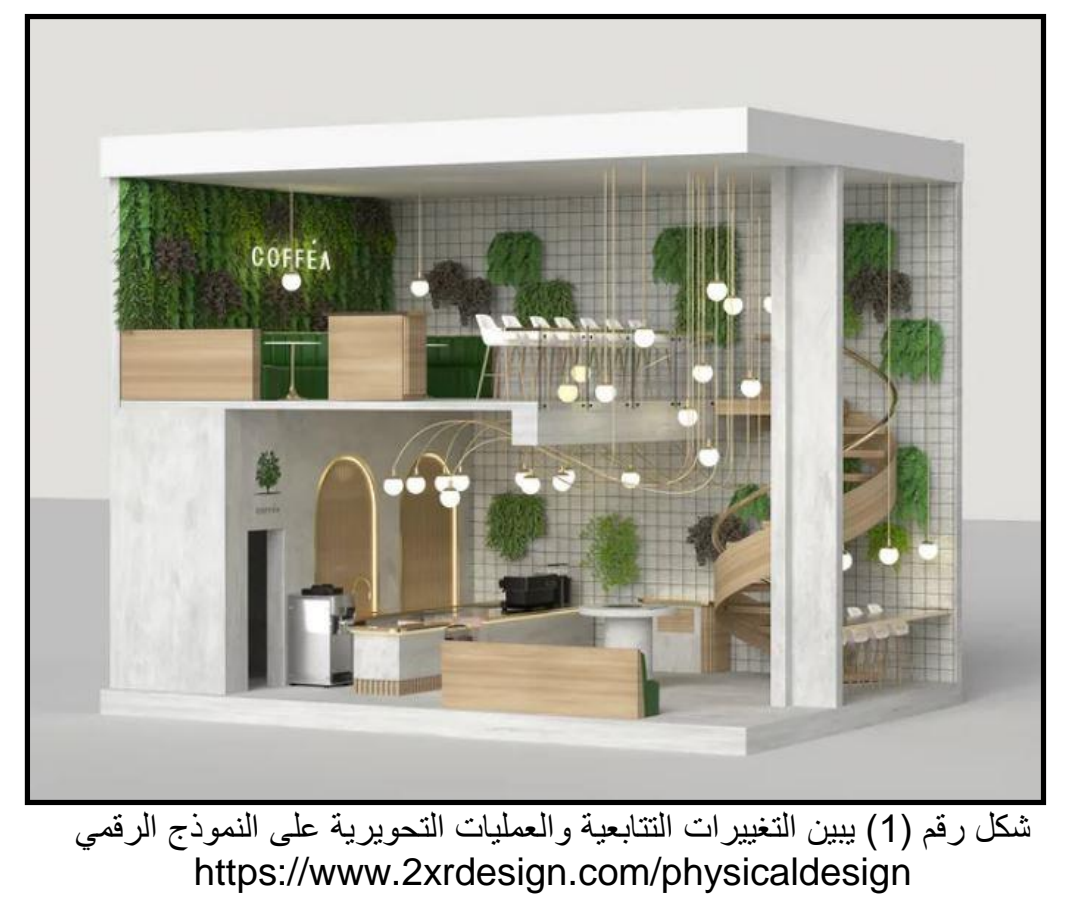

المبحث الثاني:النمذجة في التصميم الداخلي بين الواقع والمحاكاة

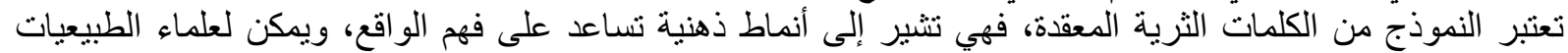

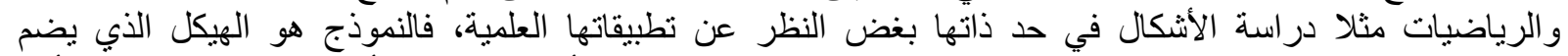

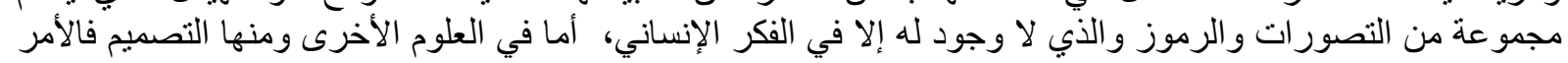

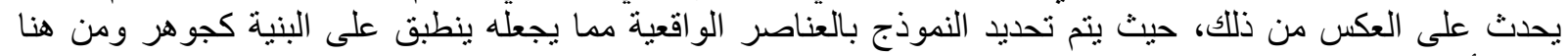

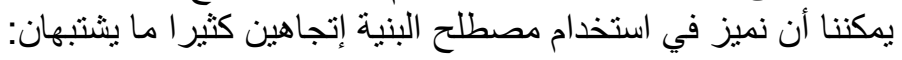

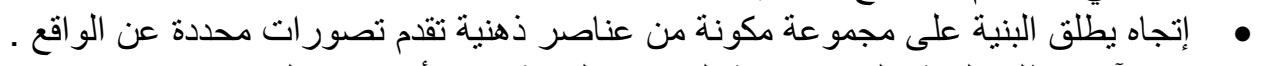

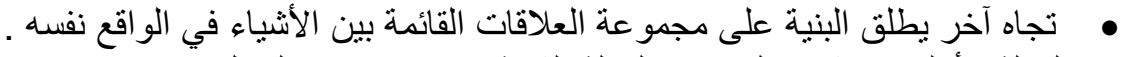
فهي في الحالة الأولى نموذج عقلي وفي الحالة الثانية واقعي كما في الثنكل(2)

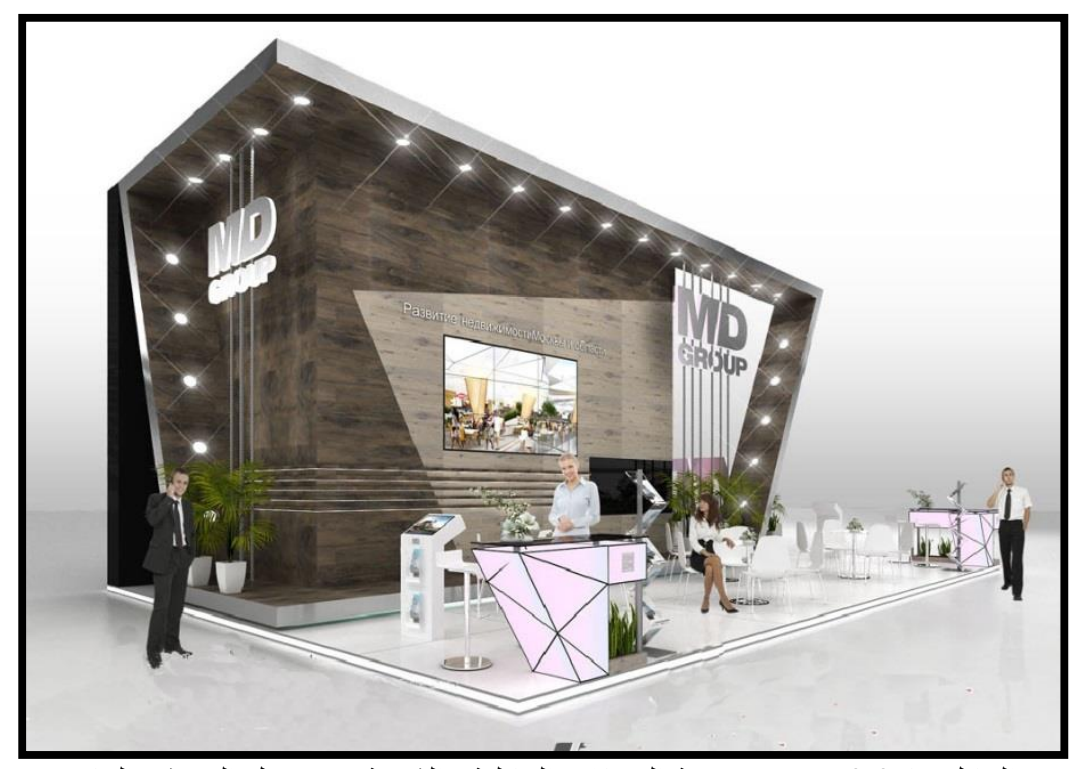

الشكل رقم(2) يبين مجمو عة العلاقات الثنكلية والذهنية من خلال النموذج الرقمي /https://www.pinterest.com/pin/597641813049850156

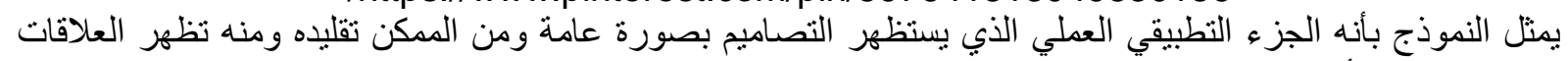

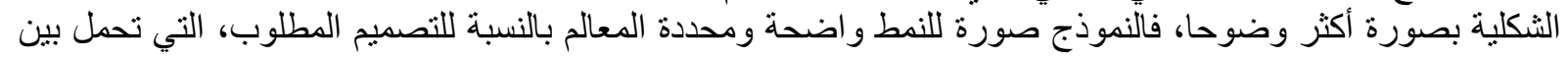




\section{DESIGN VARIABLES FOR THE USE OF DIGITAL MODELING IN CONTEMPORARY INTERIOR DESIGN}

طياتها الإبعاد الفكرية والرمزية الكامنة في العقل ويمكن تحقيقها فيزياويا بالنموذج، ونرتبط فكرة النموذج في ثلاثة

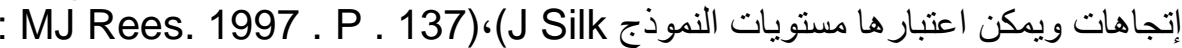

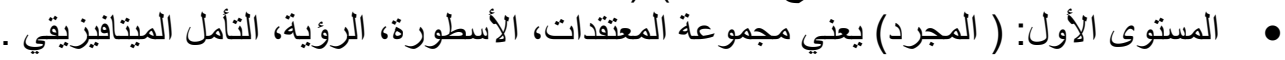

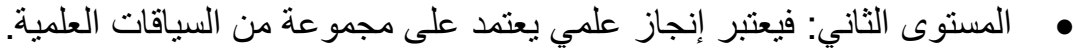

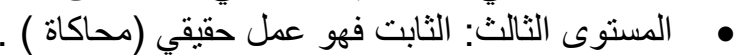

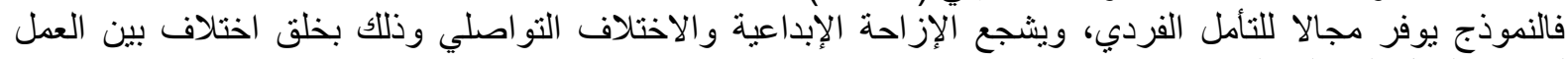

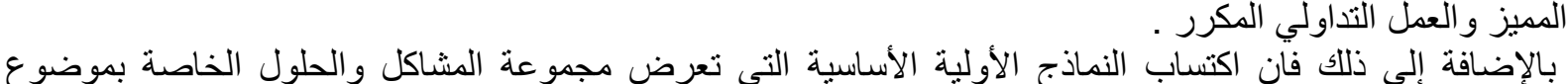

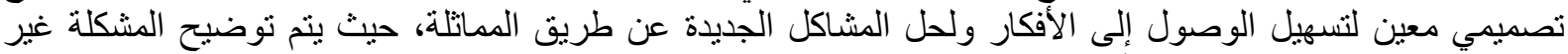

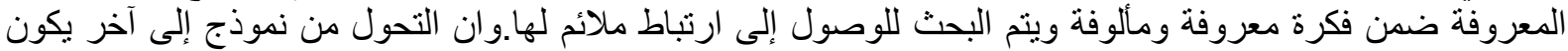

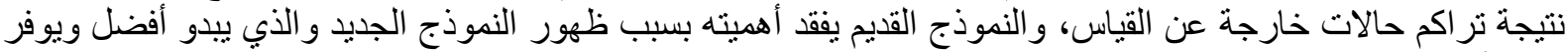
نتائج أكثر ايجابية، و هذا التحول لا يكون كاملا في البداء حيث يحتفظ النموذج الجيد بالكثير من خصائص التئ القديم النافعة ولكنه يضعه في إطار جديد بشكل جديد و ومعنى جديد.

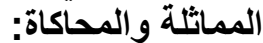

المحاكاة هي تقليد تقريبي لتشغيل العملية أو النظام،يتم استخدام المحاكاة في العديد من السياقات، مثل محاكاة التكنولوجيا

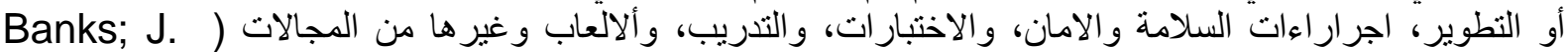

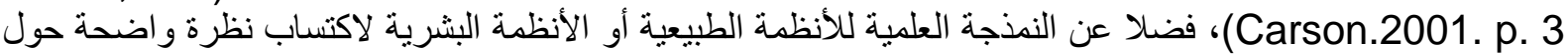

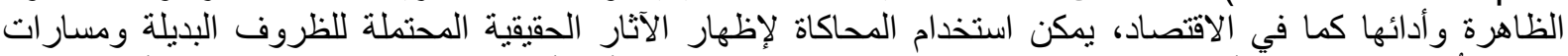

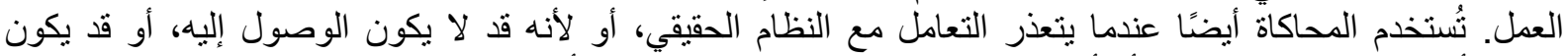

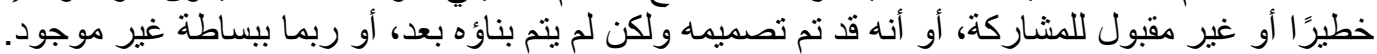

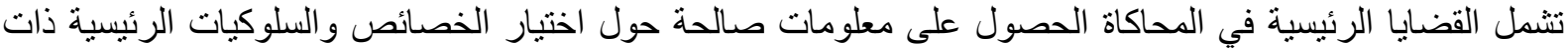

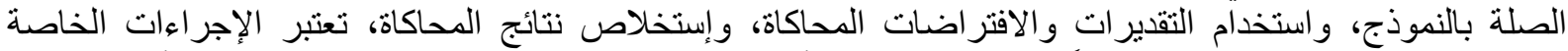

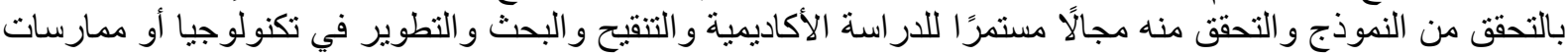

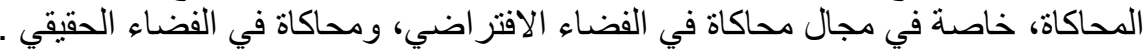

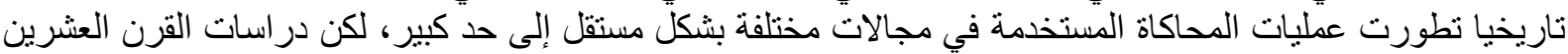

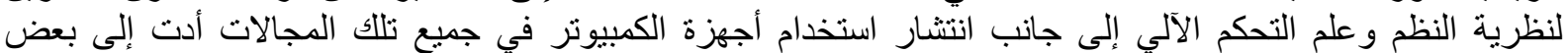

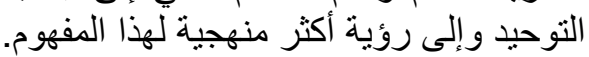

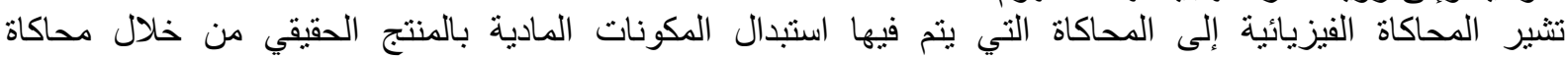

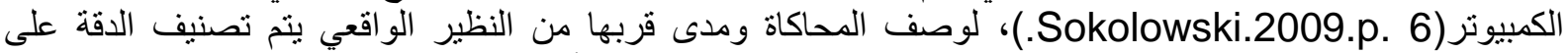

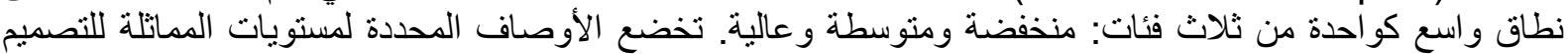

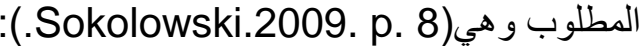
1.

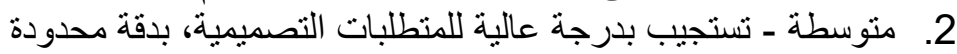

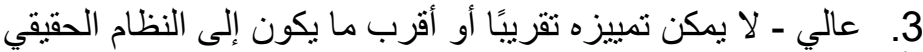

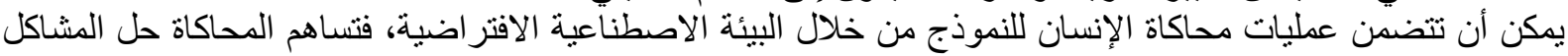
في البيئة التصميمة بفعالية كبيرة.(Bruzzone A.G.2003. 315) ، كما في الثكل (3)

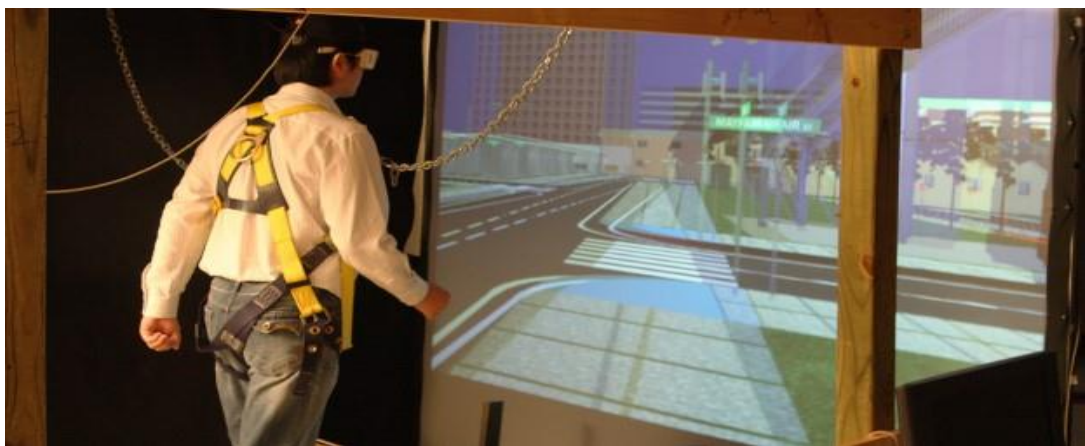

الثكل (3) يبين المحاكاة عالية من خلال الو اقع الافنراضي الانغماسي

https://www.continuuminnovation.com/en/how-we- 
أصبحت المحاكاة الرقمية جزءًا لابد منها من نمذجة العديد من النظم الطبيعية وفي الفيزياء و الكيمياء و البيولوجيا، و الأنظمة

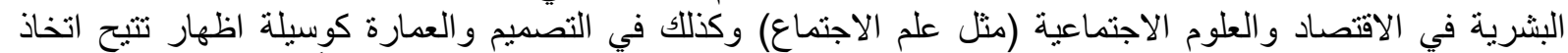

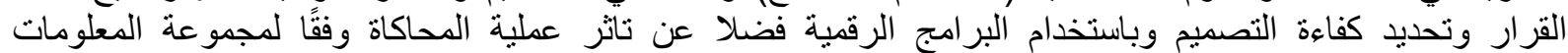
المتو افرة للبيئة التصميمية. اذ يسعى المصدم من خلال النموذج الرقمي إيجاد حلول تحليلية تتيح التنبؤ بالتصميم فضلا عن سلوك مستخدم الفضاء

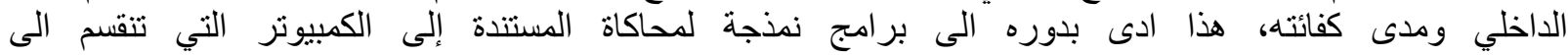
:(2008. p 86)،Davidovitch

المحاكاة "البناشرة": حيث بستخدم المصدم النموذج الفعلي بأنظمة حقيقية في بيئة حقيقية. المحاكاة "الافتر اضية": يستخدم المصمم نموذج بأنظمة محاكاة في بيئة اصطنة الصناعية.

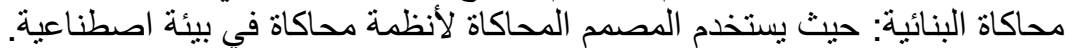
المحاكاة "الحية: يستخدم النماذج الاختبار ات المعيارية، ومن صفاتها الدقة العالية، كما في الثكل رقم (4)

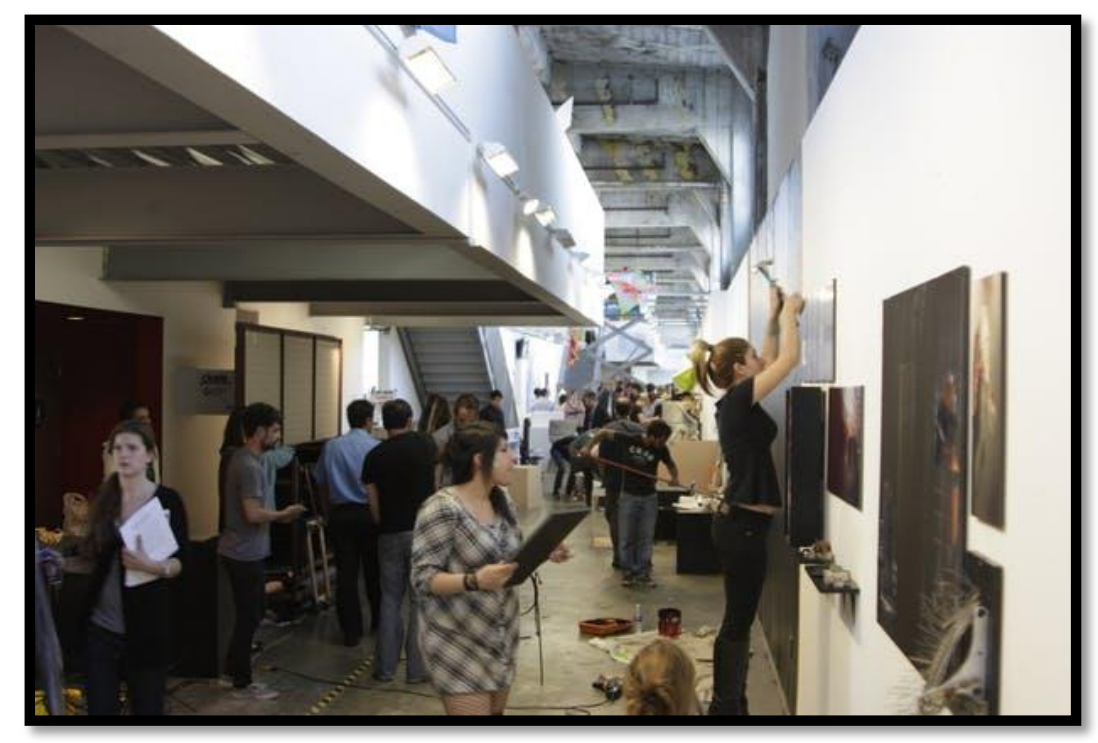

-https://archinect.com/schools/release/88/sci-arc الثكل رقم(4)يبين محاكاة البيئة الو اقعية والافنر اضية

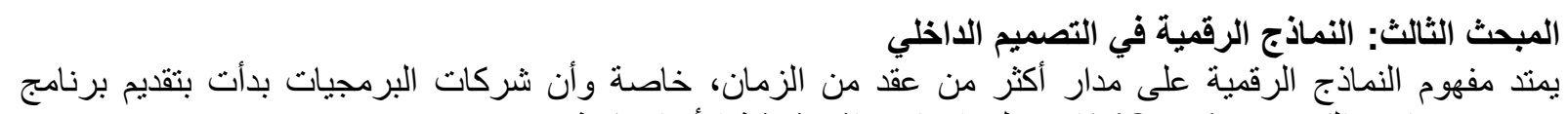

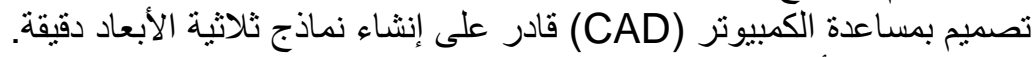

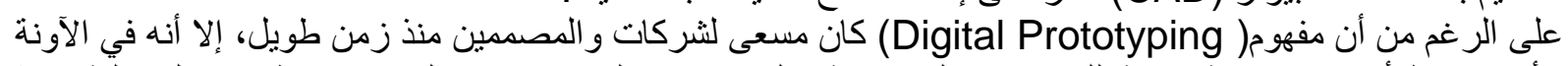

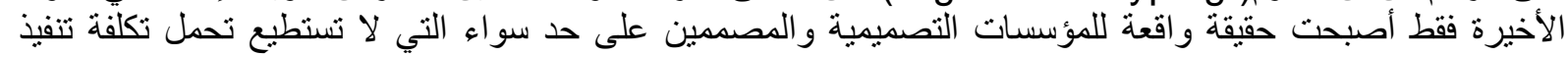
حلول معقدة ومكلفة.

النماذج الافتراضية

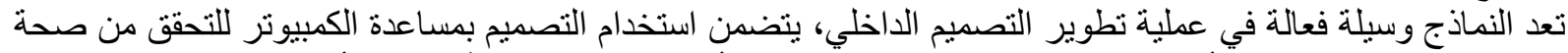
التصميم قبل اجر اء نموذج أولي مادي، يتم ذلك عن طريق إنشاء أنشال هندسية (عادةً نثلاثية الأبعاد) يتم إنشاؤها ها بواسطة

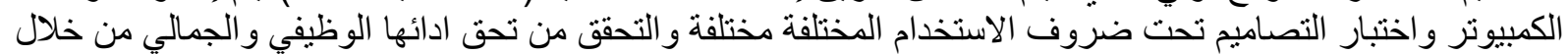

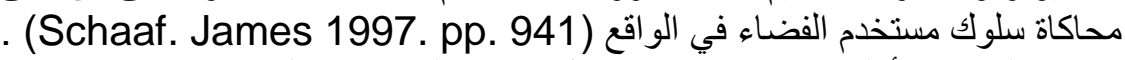

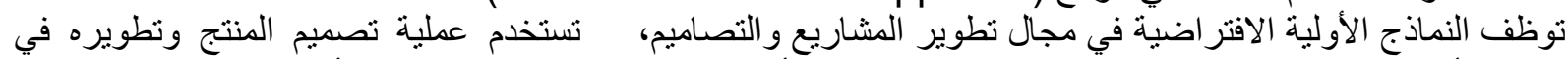

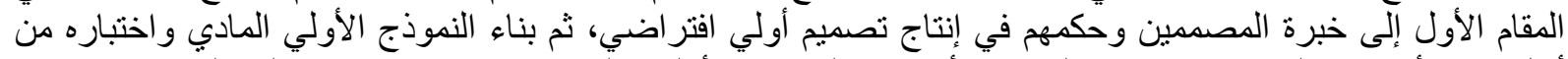

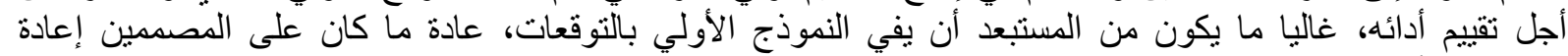

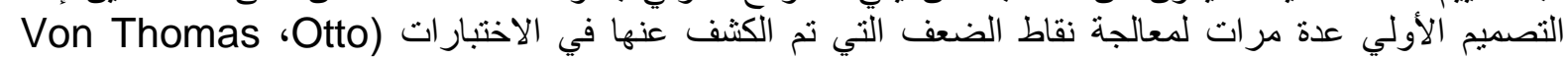

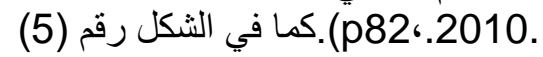




\section{DESIGN VARIABLES FOR THE USE OF DIGITAL MODELING IN \\ CONTEMPORARY INTERIOR DESIGN}

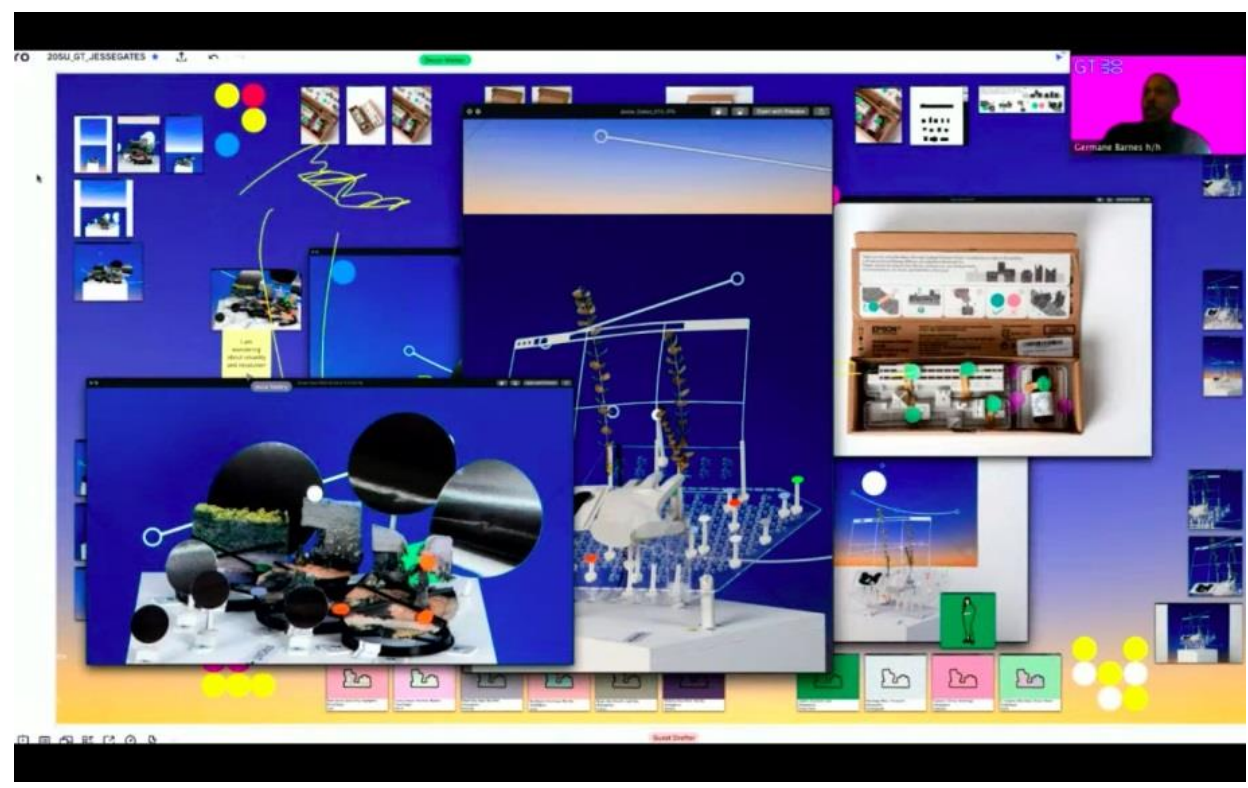

رقم(5)يبين

كل التفاصيل التي يمكن التحقق منها في آن واحد ومشاركة الاخرين

الشكل https://www.archdaily.com/948832/hga;g

تتعرض الثركات لضغوط لتقليل الوقت اللازم لتصميم وتنفيذ الفضاءات الداخلية و الوصول بها إلى مستويات أعلى من

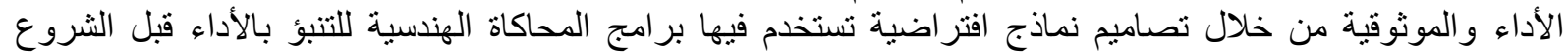

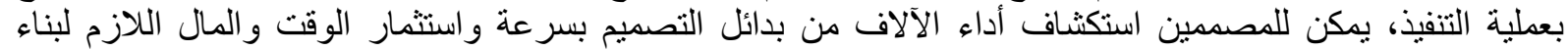

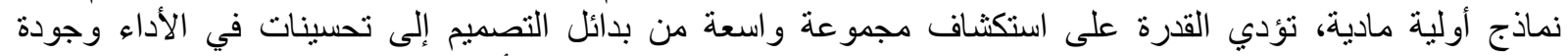

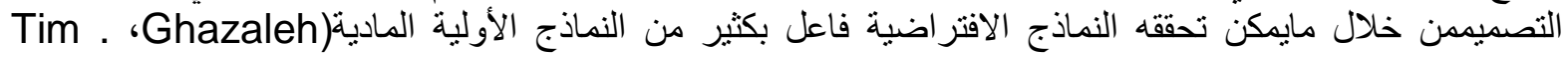
.(2004.p47 توفر النماذج الأولية صورة شاملة عن كيفية تصميم وتتفيذ الفضاء الداخلي، وهو الأبر يربط هذه العمليات بالأداء، يتيح التوافر

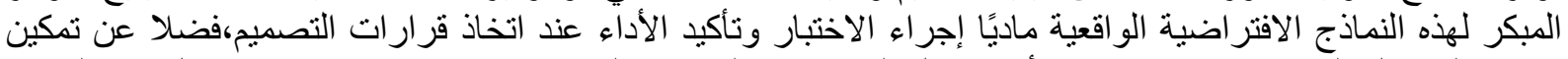

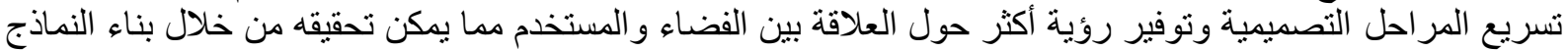

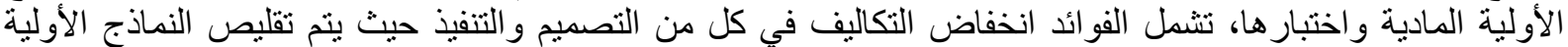

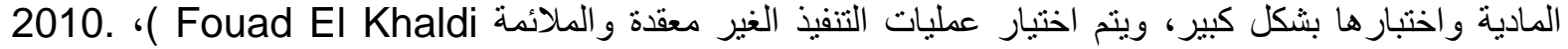
تتجاوز النماذج الرقمية مجرد إنشاء تصاميم ثلاثية الابعاد بل انها تمنح فرق تطوير التصاميم طريقة لتقييم وتقويم التصاميم بكافة كما في الثكل رقم)

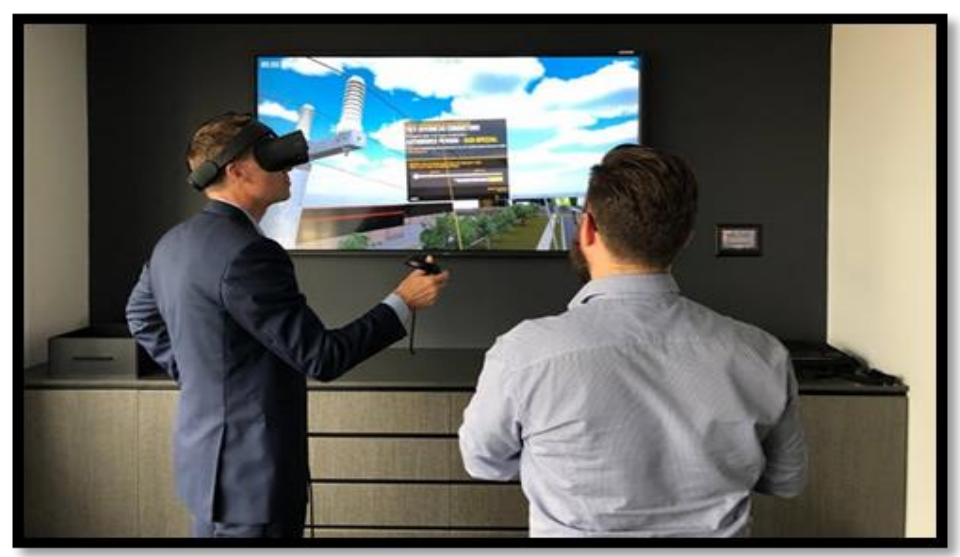

الثكل رقم(6)يبين الفريق التصميمي يقبم التصميم من خلال التقنيات الافتراضية الانغماسية /https://www.nstands.com/moscow 
عناصر ها وتفاصيلها الفضائية، لتحديد مدى موافقة التصمي مع المعايير المطلوبة،ومعرفة كيفية تفاعل مكونات التصميم التهاء

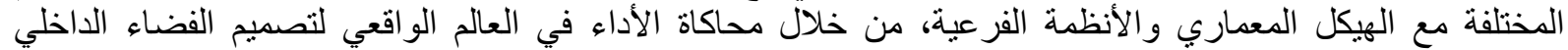

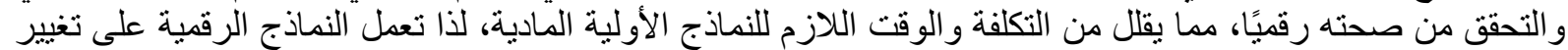
دورة تطوير المنتجات التقليدية(Jeff Row 2006.p89). يمكن للمصمم أيضًا إجراء الثئ عمليات المحاكاة في المراحل المبكرة من دورة تطوير التصميم، بحيث تتجنب أوضاع الفشل

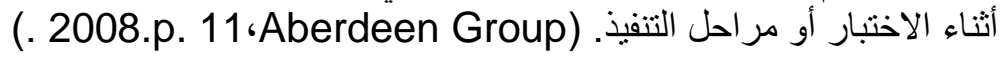

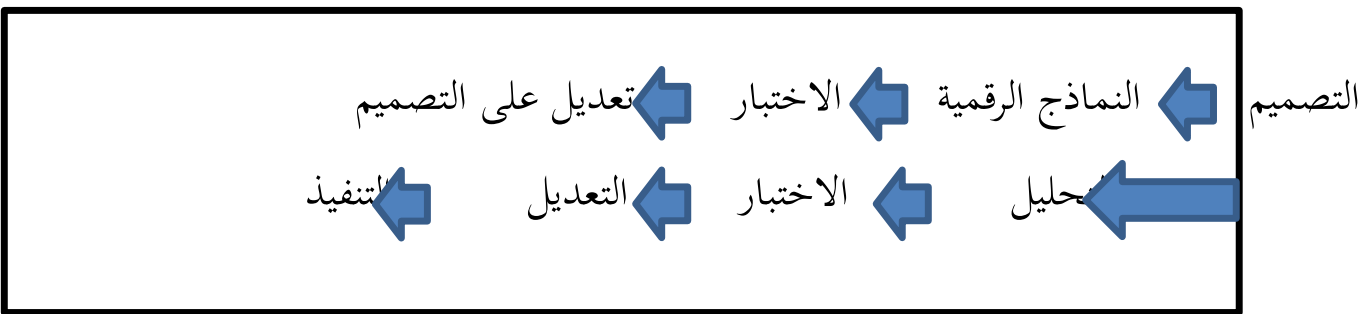

مخطط يمثل مر احل العميلية التصميمية(الباحث)

مرحلة التصميم

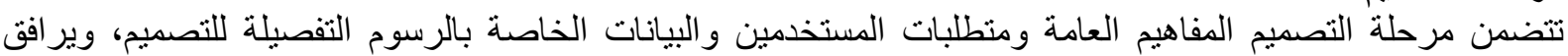

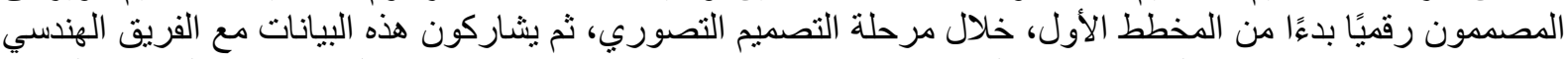

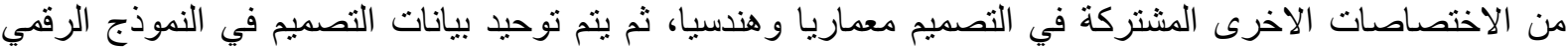
لضمان الجدوى و التقنيةكم في الثكل رقم(7).

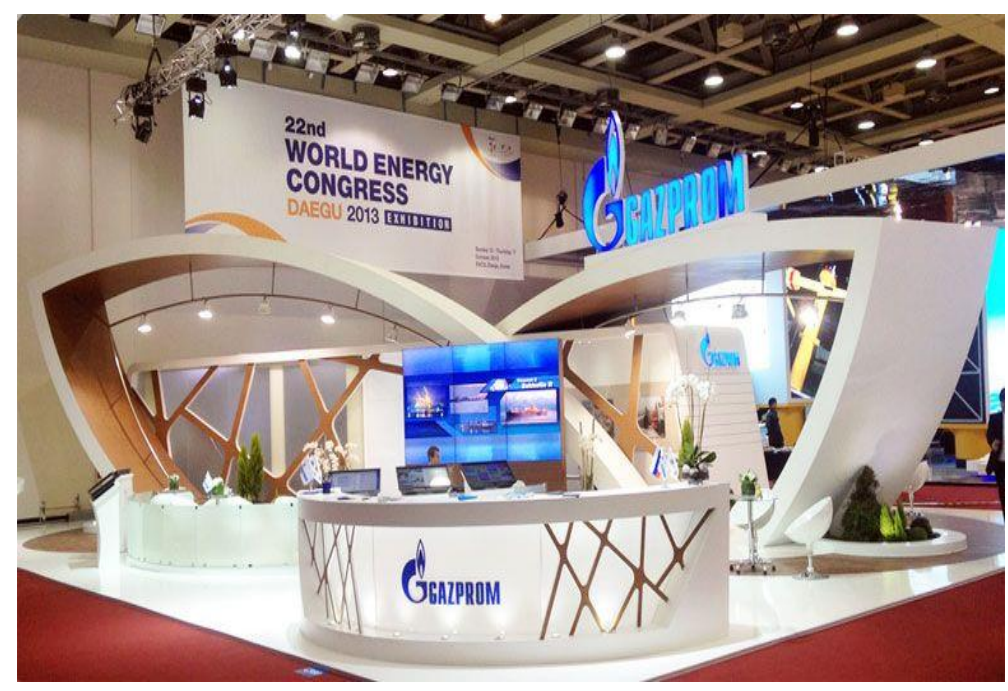

/ttps://www.pinterest.com/pin/326722147940257815 الثكل رقم(7) يبين تصميم فضاء داخلي رقميا

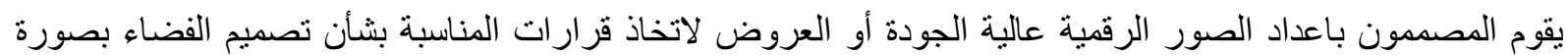

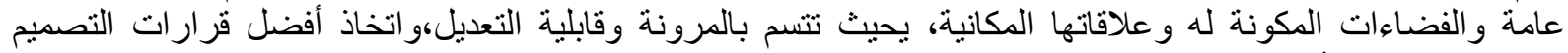
الممكنة وتجنب الأخطاء المكلفة، باستخدام النموذج الرقمي، وتوفر مرحلة تصميم النموذج الرقمي(Hallerbach، :(2018. p91 1. إجر اء حسابات متكاملة ومحاكاة الحركة في داخل الفضاء الداخلي و التحقق من صحة التحاء التصاميم.

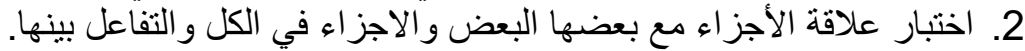

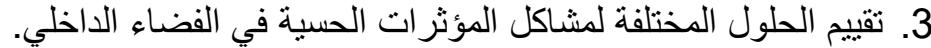

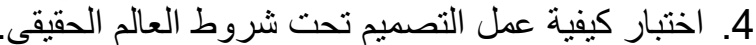
5. تحليل الإجهاد واختبار المواد المناسبة في العناصر الثكلية والفيزيائية الفضاء الداخلي. 


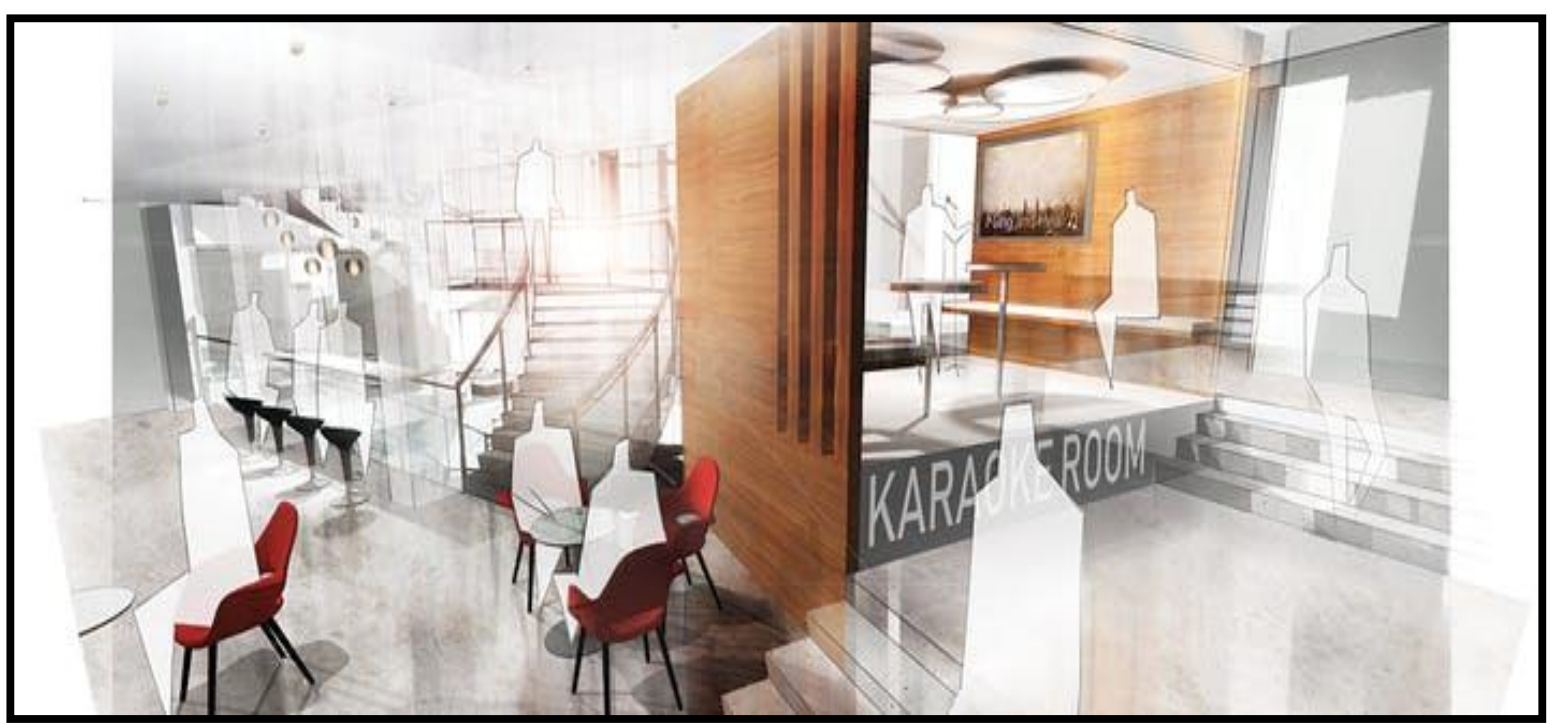

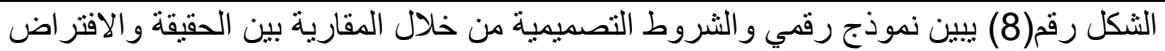
https://www.bcu.ac.uk/courses/interior-architecture-design

1. تعزز التغيرات المتسار عة والمستمرة في التغيير بشكل موازي شكليا مما يتيح خيرات غير محدة للمصمم في الابداء في تصميمه وايجاد الحلول المناسبة. 2. تتيح النماذج الرقمية للمصمم و المؤسسات المستفيدة والاشخاص القدرة على استكثاف الفضاء الداخلي قبل تصميمه.

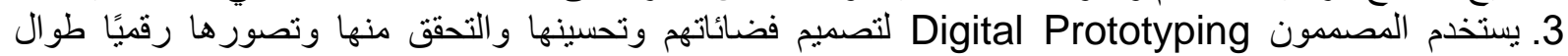

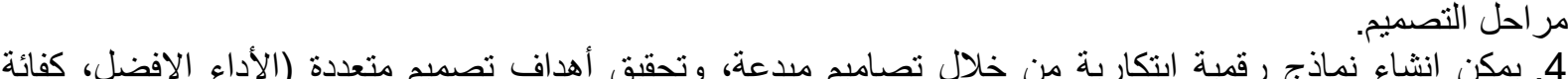
عالية، فعالية الفضاء، التكلفة المناسبة) 5. يستخدم المصممون والثركاءة فئن على حد سواء نماذج رقمية أولية لإنشاء عروض صور و اقعية ورسوم متحركة ل الفضاءات قبل تنفيذها.

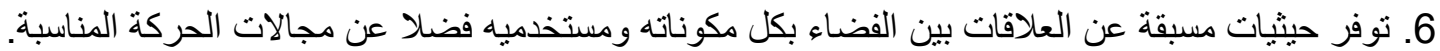

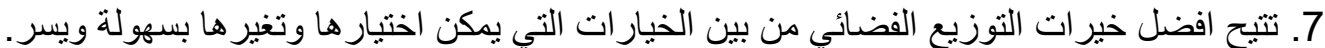

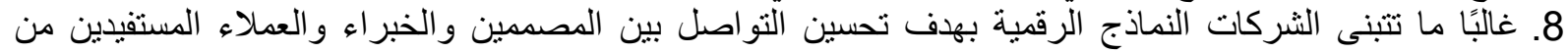

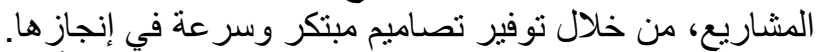

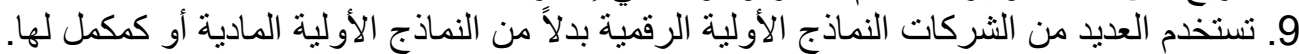

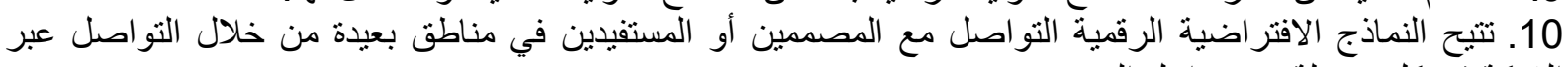
الثبكة في كل مرحلة من مر احل التصميم.

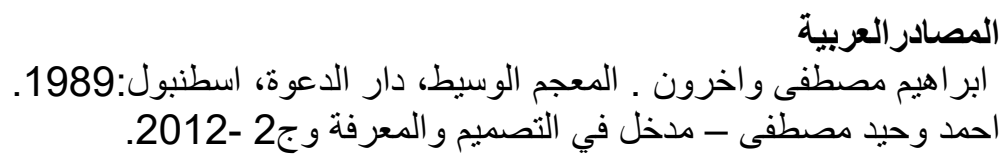
الثورة الرقمية وتانثير ها على العمارة و العمران"،المؤتمر المعماري الدولي السادي، قسم العمارة، كلية الهنسة، جامعه

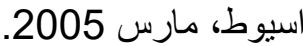

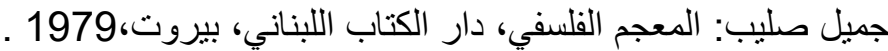

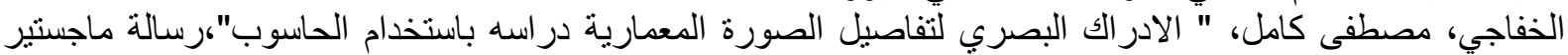

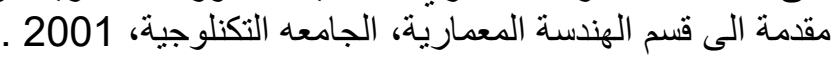

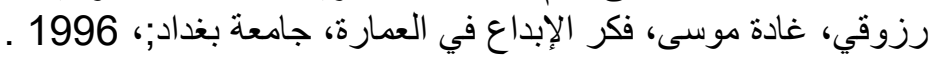
العلايلي، عبد الله:الصحاح في اللغة، دار الحضارة العربية، بيروت العية . 


$$
\begin{aligned}
& \text { لالاند، أندريه: موسوعة لالاند الفلسفية، ترجمة، خليل أحمد خليل، بيروت- باريس، منشورات عويدات، الطبعة الثانية، } \\
& 2001 \\
& \text { مجمع اللغة العربية: المعجم الفلسفي، جمهورية مصر العربية، الهيئة العامة لثئون المطابع الأميرية، 1403ه - 1983م. } \\
& \text { منير البعلبكي ـ د. رمزي منير بعلبكي، ـ المورد الحيث، } 2010 \text { ـ. }
\end{aligned}
$$

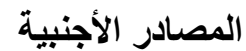

Aberdeen Group (2006). "Simulation-Driven Design Benchmark Report: Getting It Right the First Time".

.Bruzzone A.G.6 Massei M.، Simulation-Based Military Training، in Guide to Simulation-Based Disciplines، Vol.1.

Aberdeen Group، "Complementary Digital and Physical Prototyping Strategies: Avoiding the Product Development Crunch،" February 2008.

Aberdeen Group، "The Transition from 2D Drafting to 3D Modeling Benchmark Report،" September 2006.

Banks; J. Carson; B. Nelson; D. Nicol (2001). Discrete-Event System Simulation. Prentice Hall.

Campbell، Dace and Wells، Maxwell(199 )، A Critique of Virtual Reality in the Architecture .Design Process، Unvirsity of Washington، USA،

Davidovitch، L.; A. Parush \& A. Shtub (2008). "Simulation-based Learning: The Learning-Forgetting-Relearning Process and Impact of Learning History". Computers \& Education. p 866-880

Fouad El Khaldi، Raymond Ni، 2010. "Recent Integration Achievements in Virtual Prototyping for the Automo bile Industry". ESI-group.com; ESI Group. Presented.

Ghazaleh، Tim ( 2004). "Virtual Prototyping" (PDF). Printed Circuit Design \& Manufacture Magazine

Hallerbach، Sven; Xia، Yiqun; Eberle، Ulrich; Koester، Frank (3 April 2018). "Simulation-based Identification of Critical Scenarios for Cooperative and Automated Vehicles". SAE Technical Paper .

J Silk، MJ Rees، A Blanchard،( 1997) T Abel... - The Astrophysical، iopscience.iop.org،

Jeff Rowe، "Virtual Reality،" Cadalyst، 2، 2006.

Schaaf، James C.، Jr.; Thompson، Faye Lynn (2010). Systems Concept Development with Virtual Prototyping. 29th conference on Winter simulation.

https://www.2xrdesign.com/physicaldesign

$$
\text { مصادر الثبكة الدولية }
$$

https://www.archdaily.com/948832/hga;g

https://www.bcu.ac.uk/courses/interior-architecture-design-ba-

https://www.continuuminnovation.com/en/how-we-think/blog/is-your-organizationready-for-digital-prototyping>

https://www.nstands.com/moscow/

https://www.pinterest.com/pin/597641813049850156/

ttps://www.pinterest.com/pin/326722147940257815/ 